\title{
The correlation between coronal Doppler shifts and the supergranular network
}

\author{
T. Aiouaz ${ }^{1,2}$, H. Peter ${ }^{1}$, and P. Lemaire ${ }^{2}$ \\ 1 Kiepenheuer Institut für Sonnenphysik (KIS) Schöneckstraße 6, 79104 Freiburg, Germany \\ e-mail: tayeb@kis.uni-freiburg.de \\ 2 Institut d'Astrophysique Spatial (IAS), CNRS-Université Paris XI, 91405 Orsay Cedex, France
}

Received 11 October 2004 / Accepted 28 January 2005

\begin{abstract}
We examine properties of line profiles as found with large raster scans of the solar corona acquired by the UV spectrometer SUMER on board SOHO. The observed regions include an equatorial coronal hole, a polar coronal hole, as well as surrounding quiet Sun areas. In order to reveal the network and remove strong local brightenings, a filter is applied to a continuum image. The filtered continuum image, the intensity image and the dopplergram are used to produce "scatter diagrams" (dispersion plots). We find correlations between the chromospheric network, the Ne VIII (770 ̊) intensity and the Ne VIII (770 Å) Doppler shift in quiet Sun areas and in coronal holes. We establish that the maximum outflow (blue-shift) at low corona temperatures does not appear in the centre of the network but rather near network boundaries. Furthermore the maximum blue-shift seems to appear in the dark regions in Ne VIII line intensity, which is in agreement with Wilhelm (2000). The opposite correlation appears for very low intensities (less than half of the average intensity), revealing in these regions a lack of energy to either accelerate the solar wind or produce any detectable radiation. The absence of magnetic field concentration in these regions in a reconstructed magnetogram from a MDI/SOHO series seems to confirm the lack of energy.
\end{abstract}

Key words. Sun: UV radiation - Sun: corona - line: profiles

\section{Introduction}

Over the last two decades there have been numerous measurements of line shifts of the chromospheric region, the transition region and the coronal lines. The first measurements of transition region Doppler shifts were performed using the NRL normal-incidence spectrograph on-board Skylab (Doschek et al. 1976). More recently Brekke et al. (1997) and Chae et al. (1998) found red-shifts for the quiet Sun with a maximum at $1.5 \times 10^{5} \mathrm{~K}$ with a value of $11 \mathrm{~km} \mathrm{~s}^{-1}$ using instrumentation on-board SOHO (SOlar and Heliospheric Observatory). Later Peter \& Judge (1999) were the first to find blue-shifts at the disk centre for coronal lines e.g. Ne VIII (770 ̊), $\operatorname{MgX}(625 \AA)$ of about 2.6 and $4.5 \mathrm{~km} \mathrm{~s}^{-1}$ respectively over quiet Sun areas.

In this context there were many attempts to answer the question of whether Doppler shifts and intensities are correlated. Gebbie et al. (1981) using SMM data with a resolution of $3^{\prime \prime}$ found a correlation between relative red-shifts and bright regions in intensity in the C IV (1548 $\AA$ ) line. A correlation between dark regions and relative blue-shifts was found as well. These results were not confirmed by Athay et al. (1983) using SMM with the same resolution while studying Doppler shifts in the same line. Although Athay et al. (1983) noticed that a visual comparison of contour plots of Doppler shifts and intensity images shows the network to be red-shifted in quiet Sun areas, revealing the difficulty in plotting this "visual" correlation. Dere et al. (1984) confirmed the results of Athay et al. (1983) at a higher resolution of 1" using HRTS. However they also noted that some correlation was found in subsets of their data.

More recent results using SUMER on SOHO show a relationship between intensity and Doppler shift (see Brynildsen et al. 1997). Indeed Peter (1999) found the bright regions in C IV red-shifted by about $10 \mathrm{~km} \mathrm{~s}^{-1}$ while the darker parts of the internetwork show no line shifts. For Ne VIII (770 ̊) he found a net blue-shift in the quiet Sun and a sharper blue-shift in the polar coronal holes, but he did not find a correlation between Ne VIII intensity and Doppler shift on the network scalewhich was probably due to the spatial resolution and signal to noise in the dataset he used. From data obtained at the north polar region, Hassler et al. (1999) indicate that strong outflow (blue-shift) occurs along the quiet Sun network lanes especially where lanes come together. Wilhelm (2000) showed by means of contour plots that the strongest blue-shifts appear in the dark areas in the Ne VIII (770 ̊) line in a polar region. Although the correlation between Doppler shift and intensity seems to be established in C IV, it is still not clear whether coronal lines show any similar behavior, and whether characteristic differences can be found between coronal holes and quiet Sun areas and/or between equatorial and polar regions. 
Table 1. Summary of observations (data set, date, targets, line, spectral order, observation parameters, total field of view).

\begin{tabular}{clllccccc}
\hline \hline Data set & Date & Solar region & Emission line & Order & Step size ["] & Exposure [s] & Detector & FOV ["] \\
\hline 1 & March 7, 1997 & ECH and QS & Ne VIII $(770 \AA)$ & 1 & 1.0 & 90 & B & $235 \times 283$ \\
2 & September 21, 1996 & PCH and QS & Ne VIII (770 ̊) & 2 & 3.0 & 150 & A & $493 \times 283$ \\
\hline
\end{tabular}

In this context the role of the magnetic field for intensity enhancements and in velocity variations is still not clear. Kopp \& Kuperus (1968) first proposed an enhanced magnetic field as a reason for the measured intensity enhancement of UV emission lines in the temperature regime below $10^{5} \mathrm{~K}$. Later Reeves et al. (1974) observed that in lines formed above $10^{5} \mathrm{~K}$ the network structure disappears. Based on this, Gabriel (1976) developed a model relating the observed bright EUV network to magnetic flux concentrated at supergranular boundaries of the convective flows at the chromospheric level. The magnetic field expands higher up into the atmosphere through the transition region to fill the whole volume until it has become relatively uniform at the corona level. The magnetic flux then forms a funnel-like structure called a coronal funnel.

In this paper two large raster scans are presented, one of $235^{\prime \prime} \times 283^{\prime \prime}$ and one of $493^{\prime \prime} \times 283^{\prime \prime}$. These scans contain coronal holes and surrounding quiet Sun areas. We report correlation properties between the chromospheric network, coronal line intensity and Doppler shift for a polar coronal hole $(\mathrm{PCH})$, an equatorial coronal hole (ECH) and quiet Sun (QS) areas.

\section{Data analysis}

\subsection{Observations}

The EUV spectrometer SUMER was used with the $1^{\prime \prime} \times 300^{\prime \prime}$ (north-south) slit to perform the observations summarized in Table 1. The SUMER spectrograph and its first results have been described in detail in Wilhelm et al. (1995a) and Lemaire et al. (1997). The observed regions on the Sun include an ECH, a $\mathrm{PCH}$, as well as surrounding QS areas. The coronal holes were also detected in EIT Fe IX/X (see Fig. 1).

One scan of about $235^{\prime \prime} \times 283^{\prime \prime}$ was acquired in various transition region lines on March 7, 1997 (data set 1). The slit was kept at a fixed position, thus the scan was done by solar rotation over $24 \mathrm{~h}$. The observations were performed on the B detector. Windows of 50 spectral pixels (about $2 \AA$ ) were transmitted during the $90 \mathrm{~s}$ exposure time. The wavelength range includes among others the Ne VIII (770 $\AA$ ) line from the low corona. The centre of the north-south slit was pointed at solar $X=0^{\prime \prime}$ and solar $Y=279^{\prime \prime}$ in solar/SOHO disk coordinates. A more detailed description of this data set can be found in Lemaire et al. (1999). The lower right panel in Fig. 2 shows the intensity image of the Ne VIII emission line. The lower left panel in the same figure shows the dopplergram of the Ne VIII line. In both data sets the coronal hole is defined by hand using the Ne VIII (770 ̊) intensity images.

During this observation, full disk magnetograms with the Michelson Doppler Imager (MDI) (Scherrer et al. 1995) on board SOHO were performed. One full disk image was taken
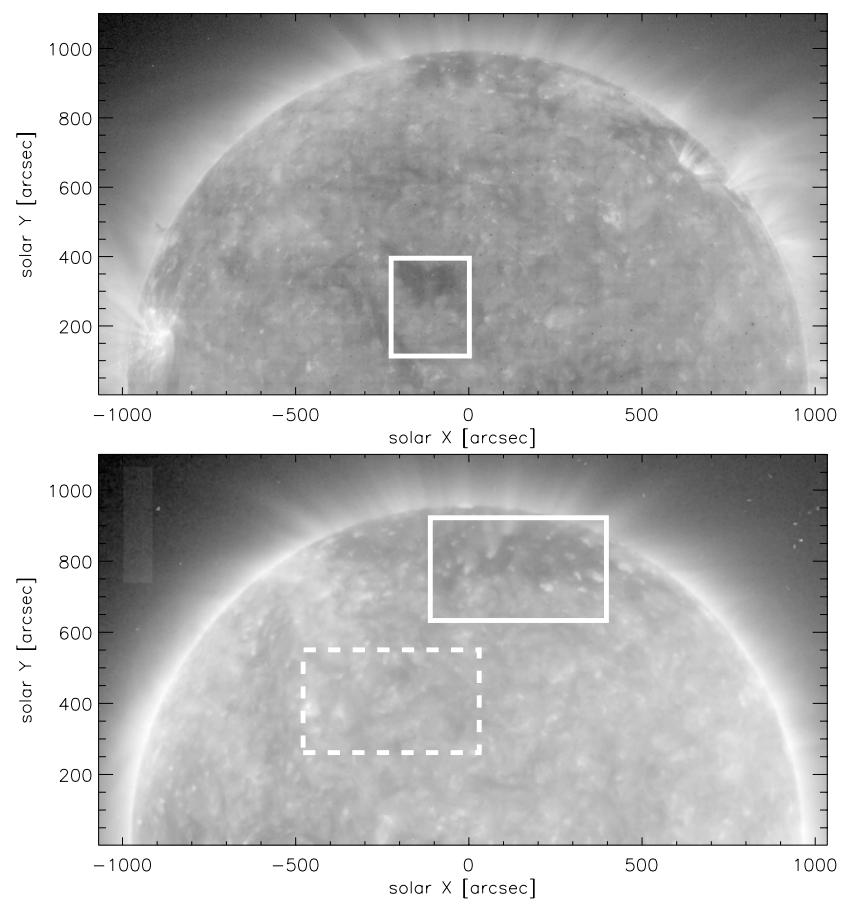

Fig. 1. EIT/SOHO images in the Fe IX/X (171 $\AA$ ) line. The areas scanned by SUMER are outlined by white contours. Upper panel: equatorial region observed on March 7, 1997. Lower panel: polar region observed on September 21, 1996. The area outlined by the dashed contour shows the observations used for the correction of data set 2 .

every $90 \mathrm{~min}$. From the MDI series (16 full disk images) we were able to reconstruct the magnetogram corresponding to the raster done by SUMER over $24 \mathrm{~h}$ with a resolution of $\sim 2^{\prime \prime}$ (see upper right panel in Fig. 2). The area covered by the SUMER slit during the $24 \mathrm{~h}$ raster scan was compiled from individual slices of the corresponding magnetograms from the MDI series. The resulting magnetogram is pieced together in order to correspond, as closely as possible, to the SUMER raster scan. One has to keep in mind that the magnetogram reconstruction introduces a misalignment to the intensity images of a few pixels due to the time resolution limit. A variation with time of the signal-to-noise ratio can be observed in the MDI series. Thus the reconstructed magnetogram shows a varying noise level. This variation is well-known and documented in the "MDI Calibration Notes and Known Problems" section on the MDI web-site (http://soi.stanford.edu/data/cal). Since we are only interested in the position of the strong magnetic field concentration, there is no need to correct this pattern.

Another raster scan of about $493^{\prime \prime} \times 283^{\prime \prime}$ was done on September 21, 1996 (data set 2) centered at solar $X=246^{\prime \prime}$ and solar $Y=781^{\prime \prime}$. This data set has been studied by Hassler et al. (1999) and Wilhelm (2000). The scan was taken with a step size of $3^{\prime \prime}$ and a sampling time of $150 \mathrm{~s}$. The central 

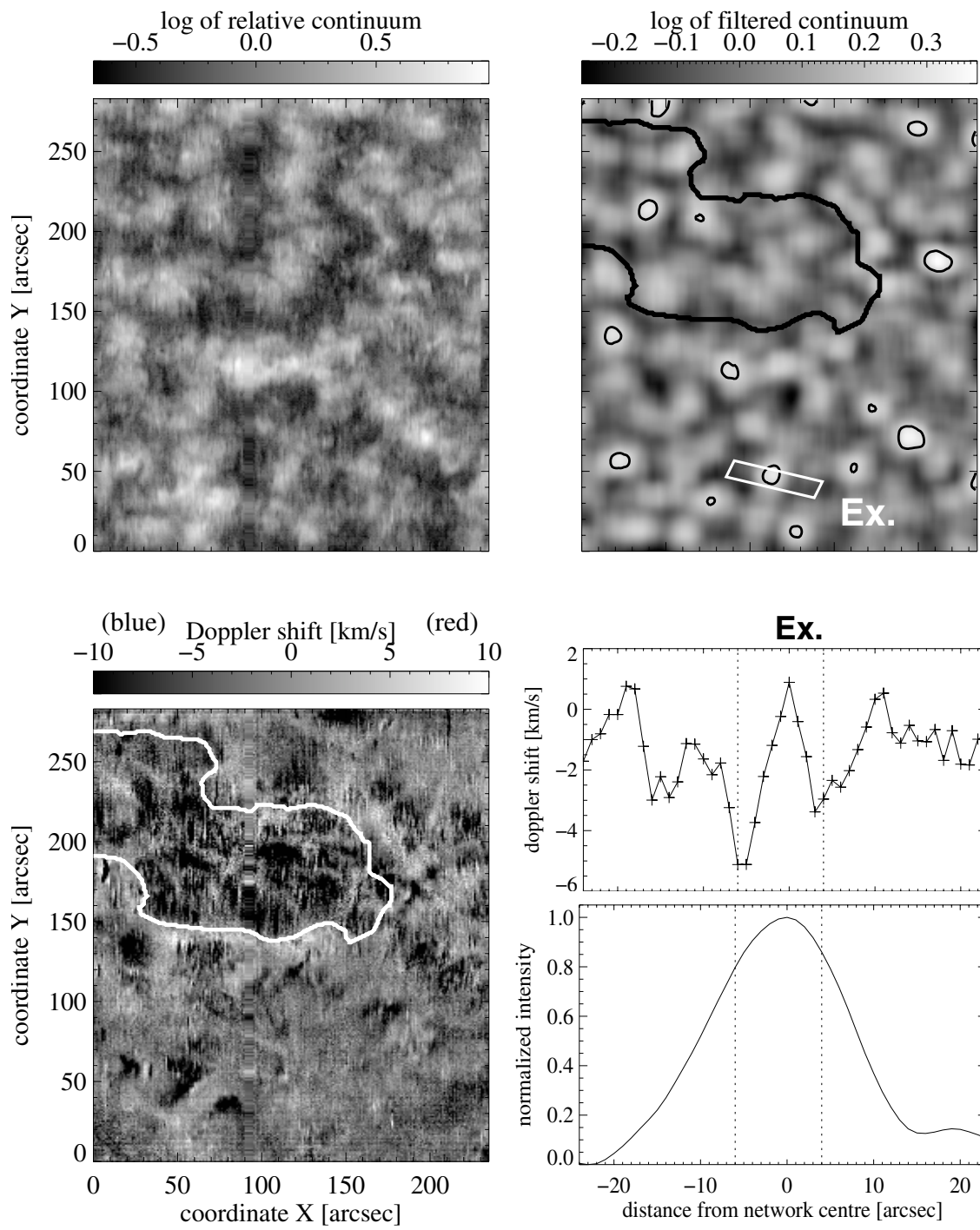

Ex.

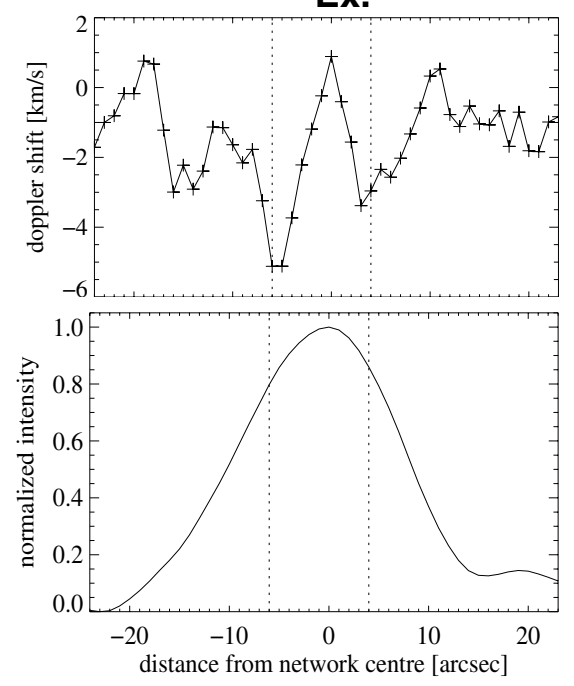

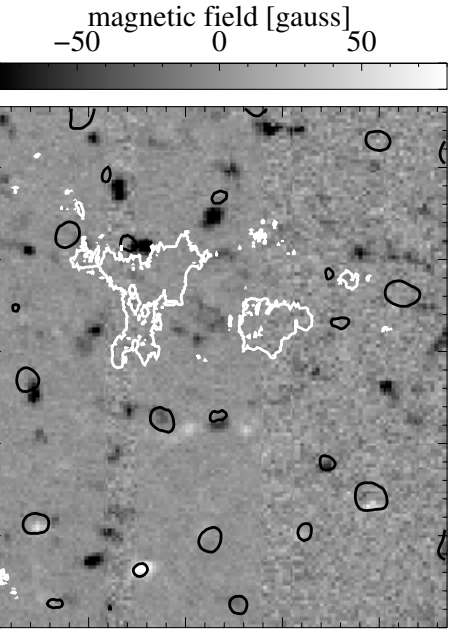

log of relative intensity

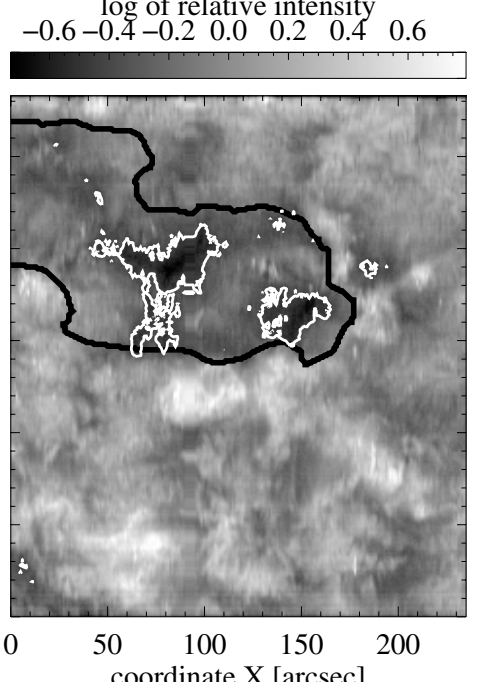

Fig. 2. Data set 1 . The Coronal hole is outlined by a thick solid line on the filtered continuum image, the Dopplergram and the Ne VIII intensity image. Upper left: continuum intensity image taken with SUMER/SOHO on logarithmic scale. Middle: filtered version of the left image with contour plot $\log (I /\langle I\rangle)=0.25$ in thin black solid line. Right: reconstructed magnetogram from data taken with MDI/SOHO. The thin contour plots of the Ne VIII intensity image and the filtered continuum image are overplotted on this image. Lower left: Dopplergram of the Ne VIII (770 ̊) line observed on March 7, 1997. Middle: the line shift across the network from cell centre to cell centre, i.e. along the long edges of the box, is shown in the upper panel for the box "Ex.". Right: Ne VIII line intensity image with contour plots of $\log (I /\langle I\rangle)=-0.40$ in thin white solid line.

portion of the detector A containing the Ne VIII (770 ̊) line was read out and 512 spectral pixels were transmitted to the ground.

\subsection{Data reduction}

Both SUMER data sets were processed using the standard SUMER procedures: flat-field, geometrical distortion and dead-time correction. In order to increase the signal-to-noise ratio on the first data set, we averaged the data over 4 exposures in time (or $6 \mathrm{~min}$ ), resulting in spatial maps with pixel sizes of about 0.95 " in the scan direction (solar $X$ ). Although the standard procedures provide good first-order corrections it is necessary to remove spurious line shifts due to the geometric distortion of the detector image (see Peter \& Judge 1999).
The data set taken on March 7, 1997 shows a periodic drift due to thermal deformation of the instrument and thus a trend of the line position within the $24 \mathrm{~h}$ scan. Despite the very high thermal and mechanical stability characterizing the instrument, a drift of the whole spectral image has been detected by Curdt et al. (1997) and later analyzed by Peter \& Judge (1999). A periodic drift with an amplitude up to 1 pixel and a periodicity of $2 \mathrm{~h}$ is reported in the previous cited publications. A smoothing procedure was applied to the mean line position in order to remove residual variations of solar origin, obtaining a good estimation of the large-scale instrumental drift. The data were then corrected for this drift.

The data set taken on September 21, 1996 scanned the north pole of the Sun. In order to properly correct instrumental effects on this data set, data from a supplementary observation taken 
the day after was used to estimate the large-scale instrumental drift. The observational settings were identical to those on September 21, 1996 except that the region observed was only quiet Sun (see dashed contour in lower panel in Fig. 1). The correction of the geometrical residual is done similarly as for data set 1 .

Peter (1999) found the centre-to-limb variation of Doppler shifts to be consistent with predominantly radial flows. The centre-to-limb variation becomes significant for heliocentric angles $\theta$ bigger than $60^{\circ}$. This pattern is described for the data set 2 in detail by Dammasch et al. (1999). To allow a comparison between the polar data and the equatorial ones, we correct for the centre-to-limb projection effect. A $\cos \theta$ normalization in the form introduced by Peter \& Judge (1999) was applied to Doppler shift values located at heliocentric angle $\theta$ bigger than $60^{\circ}$. A map of the Doppler shifts in the polar region corrected in this way is shown on the lower panel of Fig. 3. Another effect due to the large heliocentric angle appears in intensity images. Due to foreshortening the supergranular cells seem to shrink with increasing heliocentric angle. The network seems thus compressed at the limb and the intensity increases toward the limb.

SUMER has a velocity sensitivity of $\sim 2 \mathrm{~km} \mathrm{~s}^{-1}$, and even $1 \mathrm{~km} \mathrm{~s}^{-1}$ for strong enough lines as shown with simulation studies in Wilhelm et al. (1995b). Similar sensitivities have been obtained under operational conditions by e.g. Warren et al. (1997), Judge et al. (1998) and Peter \& Judge (1999). In order to perform reliable Gaussian fits of the various lines we used a very robust and reliable forward-fitting technique driven by a Genetic Algorithm (PIKIA software) described in Charbonneau (1995).

\section{How to define the chromospheric network}

Various methods can be found in the literature to extract the chromospheric network. Most approaches so far use intensity threshold values to divide intensity images into network/internetwork structures. These thresholds can be defined in different ways (see Reeves 1976; Gallagher et al. 1998).

Since our aim is not only to outline the network patches but also to find characteristics of the transition from network to internetwork, it is crucial to outline the chromospheric network without distorting the distribution (histogram) of the continuum image. Thus a method is introduced which filters local brightenings and conserves the global distributions of the image. Pauluhn et al. (2000) tested different functions to fit radiance distributions (double Gaussian, lognormal) on very large data sets of radiance measurements of the quiet Sun near solar disk centre taken with CDS on board SOHO. They showed that the distribution was best fitted by a lognormal distribution rather than a double Gaussian. Earlier, Griffiths et al. (1999) found similar results with SUMER observations. We analyzed Ne VIII line as well as continuum intensity distributions which all appear close to lognormal (Gaussian in $\log I$ ) as seen in Fig. 4. However, the upper row of Fig. 4 which represents filtered continuum intensities show distributions that deviate slightly from Gaussian ones (in $\log I$ ). This deviation results from the separation of the coronal hole from the quiet Sun areas
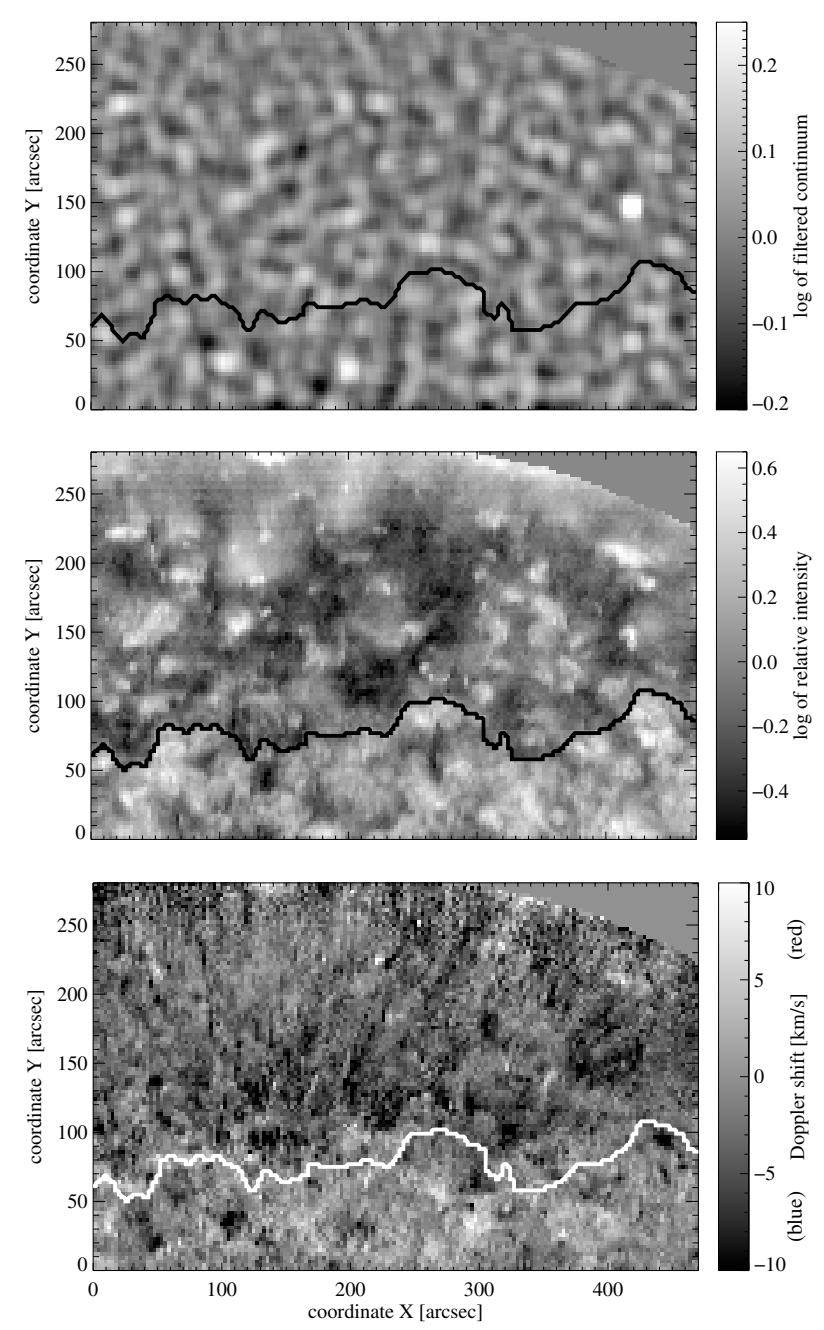

Fig. 3. Data set 2. The polar coronal hole is outlined by a solid line. Values outside the disc are set to zero. Upper: filtered continuum intensity image on logarithmic scale computed from the continuum intensity taken with SUMER/SOHO. Middle: Ne VIII (770 ̊̊) line intensity taken with SUMER/SOHO. Lower: Dopplergram of the Ne VIII (770 ̊) line observed on September 21, 1996 corrected for the centre-to-limb variation.

although coronal holes cannot be seen in the filtered continuum images. This operation results in an artificial separation of the lognormal distribution of the full image in two parts slightly deviating from Gaussian (in $\log I$ ).

The method chosen here to reveal the network involves no threshold value which arbitrarily separates the network from the internetwork. In order to point out the chromospheric network a continuum image is chosen (see upper left panel in Fig. 2). To reveal the supergranulation on the continuum image, one has to remove the local brightenings and thus reduce the contrast. We carried out a median filter of the continuum. The width of the median filter was chosen according to the approximate chromospheric supergranular cell width of $30^{\prime \prime}$. The original image is then divided by the median filtered one. Finally since we want the network centre to correspond to the intensity maximum across the network, the resulting image is smoothed over $10^{\prime \prime}$ which corresponds to the usual accepted value for the chromospheric network width (Patsourakos et al. 1999). The 


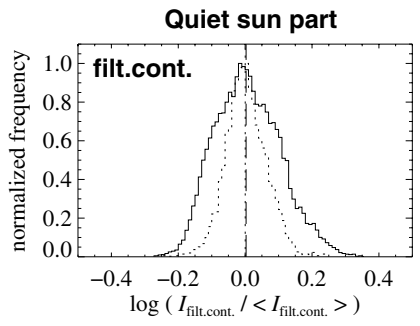

Coronal hole part

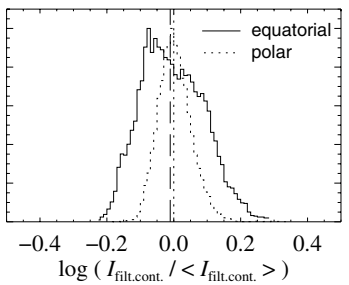

$\log \left(I_{\text {filt.cont. }} /<I_{\text {filt.cont. }}>\right)$
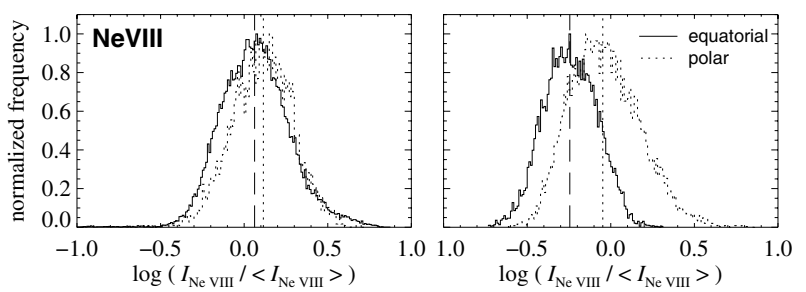

Fig. 4. Solid lines refer to the equatorial region, while dotted lines refer to the polar region. Each region is separated in two parts: quiet Sun and coronal hole. The vertical dashed lines give the median value of the corresponding histogram. Upper panels: histogram of filtered continuum image. Lower panels: histogram of the Ne VIII line intensity image.

smoothing and the median filtering were processed using the SMOOTH and the MEDIAN function in the IDL ${ }^{\mathrm{TM}}$ software. The resulting filtered continuum is shown in the upper middle panel in Fig. 2.

These operations reveal the supergranulation network without overly affecting the global distribution of the filtered continuum which remains lognormal. This filtering thus allows one to distinguish between network centre, network boundaries and internetwork. Another interesting aspect of this method is the cell-like pattern of the supergranular network appearing in the filtered continuum image, a pattern which is very difficult to achieve using a "threshold" method. The same method was used on the second data set (see upper panel in Fig. 3).

\section{Observational results}

The discussion of the observational results concentrates on two relations: (1) The relation of Ne VIII Doppler shifts ( $\left.v_{\mathrm{D}}\right)$ vs. the filtered continuum emission $\left(I_{\text {filt.cont. }}\right)$. This highlights the connection between the chromospheric (magnetic) structure and the dynamics in the corona. (2) The Doppler shift of the $\mathrm{Ne}$ VIII $\left(v_{\mathrm{D}}\right)$ but now compared to the total emission $\left(I_{\mathrm{Ne} \text { VIII }}\right)$ of that same coronal line. This relation contains information on the coronal energy balance: how much of the energy flux into the corona is transformed into acceleration and how much into heating of the plasma, finally setting the coronal pressure and thus the emission in coronal lines $\left(I_{\mathrm{Ne} \text { VIII }}\right)$.

Therefore the relations $v_{\mathrm{D}}$ vs. $I_{\mathrm{NeVIII}}$ and $v_{\mathrm{D}}$ vs. $I_{\text {filt.cont. }}$, described in dispersion plots (see below), provide insight into the energetics of the corona and its connection to the chromosphere.

\subsection{Coronal Doppler shift maps}

The Ne VIII (770 ̊) line shift images are displayed in the lower left panel in Fig. 2 for the equatorial region and in the lower panel in Fig. 3 for the polar one. Negative values in Doppler shift correspond to blue-shift, positive to red-shift. To obtain absolute values, the mean value of the Doppler shift over the whole quiet Sun was set to $-2.5 \mathrm{~km} \mathrm{~s}^{-1}$ for both data sets, as derived by Peter \& Judge (1999). Then the Doppler shifts above the limb are zero, as expected for an optically thin emission line.

The Doppler shift maps clearly outline the coronal hole area, and in the quiet Sun areas we can see some supergranular structures. However, this visual impression is hard to quantify. Even the average Doppler shift of the on-disk coronal hole (lower left panel in Fig. 2) differs only slightly from the quiet Sun part $\left(-4.4\right.$ vs. $\left.-2.1 \mathrm{~km} \mathrm{~s}^{-1}\right)$, even though the eye immediately identifies the coronal hole. In the following we will discuss how to quantify the network vs. internetwork properties.

\subsection{Dispersion plots: Doppler shift vs. intensities}

To study the relation of the line and filtered continuum intensities with respect to the Doppler shifts we binned the intensity images and calculated the median value of the Ne VIII Doppler shift in each intensity bin. The respective histograms of the intensities are shown in Fig. 4. We have normalized the intensities with respect to the median value to allow a comparison between the equatorial region (data set 1 ) and the polar region (data set 2) by using $\log (I /\langle I\rangle)$, where $I$ is the mean intensity of the corresponding bin and $\langle I\rangle$ is the median value of the intensity over the whole image.

The median Doppler shifts of the respective intensity bins are plotted vs. the normalized intensities in Figs. 5 and 6. While Fig. 5 shows the Ne VIII Doppler shift as a function of the filtered continuum intensity, Fig. 6 shows the Ne VIII Doppler shift vs. the Ne VIII line intensity. In the following we will call these diagrams dispersion plots.

Each dispersion plot has been fitted with a linear function to emphasize the trend. The slope " $a$ " is given for each fit. The standard deviation $\sigma$ of the sample of $N$ data points in the respective intensity bin is plotted as shaded areas on the dispersion plots. The minimum number of data points per bin was chosen as $N_{\min }=20$. Intensity bins with fewer data points were discarded. This ensures that the standard error of the median on each bin $\langle\sigma\rangle / \sqrt{N}$ is kept smaller than $1 \mathrm{~km} \mathrm{~s}^{-1}$. The number of data points per bin rises to $\sim 2000$ points at intermediate intensities. The sampling steps of the intensity bins were approximately 0.03 for the Ne VIII intensity plots and 0.01 for the filtered continuum ones. These steps change slightly from one plot to another since the number of points per plot varies. This variation is due to the requirement of having at least 20 values for any particular bin.

\subsection{Doppler shift vs. filtered continuum intensity}

Figure 5 shows the Doppler shift as a function of the filtered continuum intensity. The top row describing the data from the equatorial region shows a systematic variation with a pronounced maximum blue-shift. 


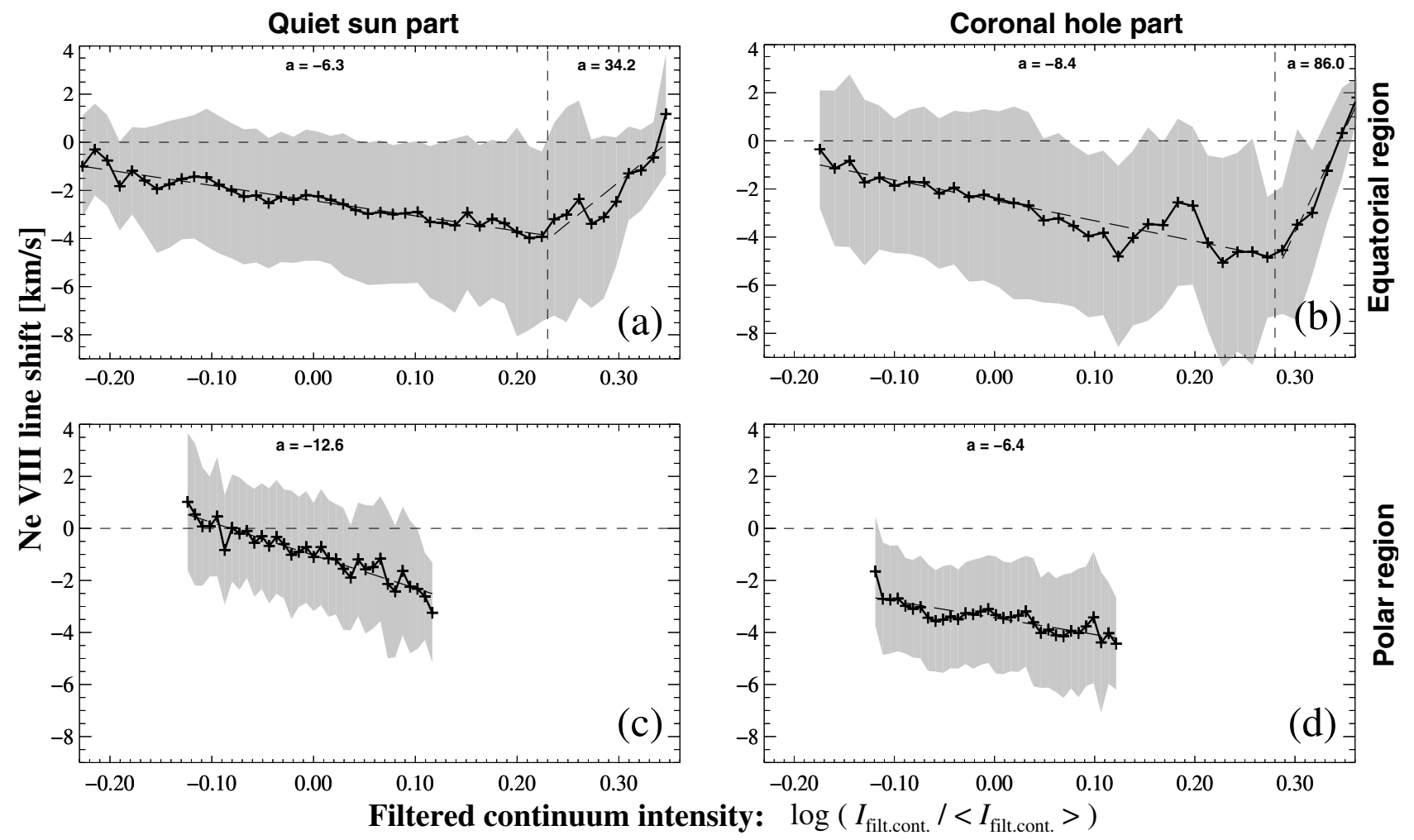

Fig. 5. Dispersion plots showing the relation between filtered continuum intensity and Ne VIII Doppler shift, in solid lines. The dispersion plots are fitted by linear fits in long dashed lines, where $a$ is slope of the fit. The left column shows quiet Sun data, the right column coronal hole data. In the first row data from the equatorial region were used, the second row describes the data from the polar region. The standard deviation of the median value is represented by the shaded areas. The vertical long-dashed lines show the position in the dispersion plot where the maximum blue-shift is reached.
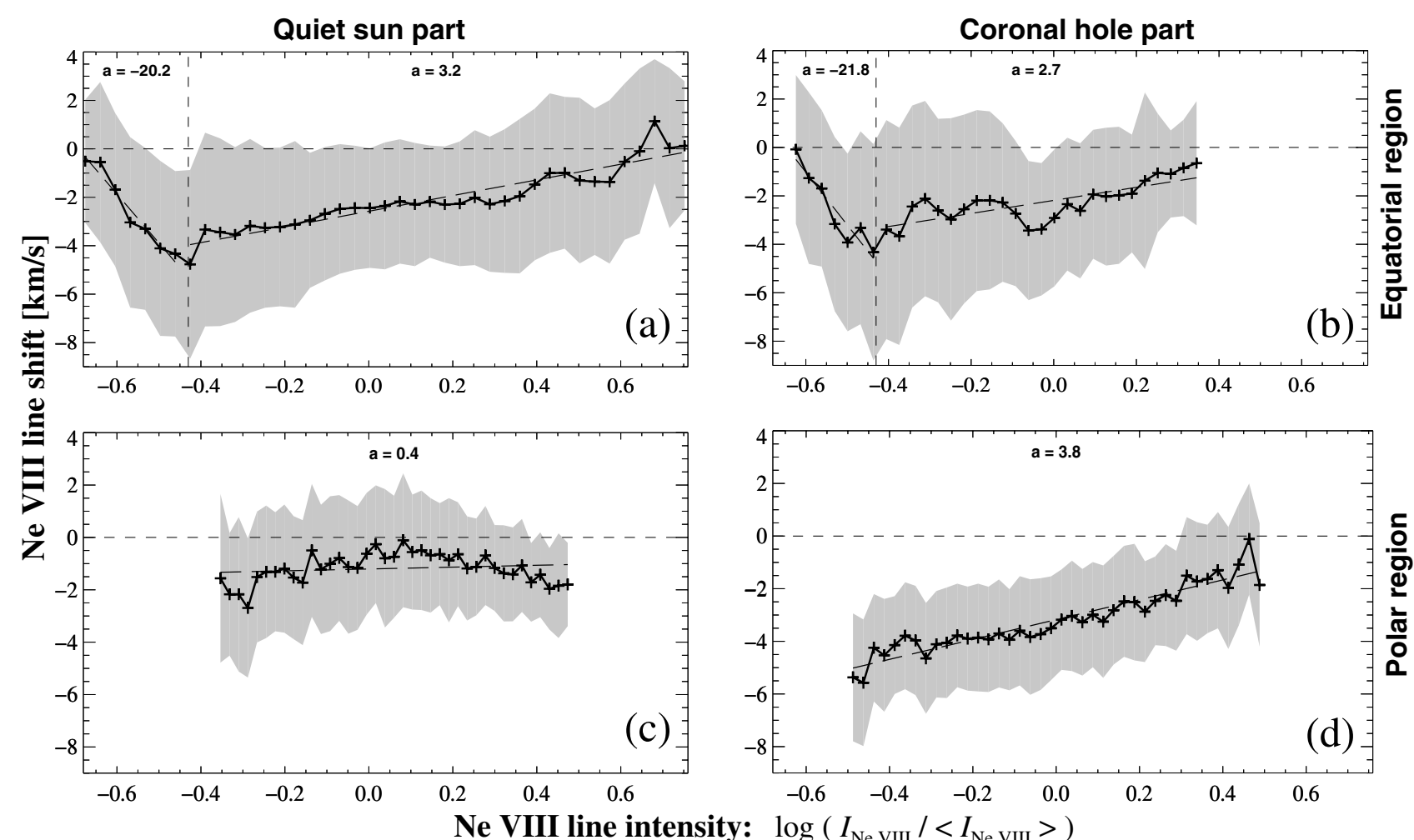

Fig. 6. Same as Fig. 5, but relating the Ne VIII line intensity to line shift. 
The main part of the dispersion plot shows a clear increase ( $a=-6.3$ for quiet Sun and -8.4 for coronal hole) of the blueshift with increasing filtered continuum intensity. In contrast, the trend for the highest intensities is the opposite: a strong decrease of the blue-shift is observed for increasing filtered continuum. Except for the absolute values of the slopes no fundamental differences are observed between quiet Sun and the coronal hole, although the maximum blue-shift appears for quiet Sun areas at $\log (I /\langle I\rangle)=0.23$ and at 0.28 for the coronal hole data. At the brightest patches of the network the Doppler shift of the coronal line tends to be zero.

The polar region data (Fig. 5 lower row) show the same behavior as the main part of the dispersion plot of the equatorial data although some differences are observed. Firstly the polar data contrast is lower than the equatorial one. This difference can also be observed in the histograms in Fig. 4 and can easily be explained. Since the heliocentric angle is larger for the polar region than for the equatorial one, the structures in the polar region are crushed, which results in a smoother distribution. The slope is steeper for the quiet Sun $(a=-12.6)$ than for the polar coronal hole $(a=-6.4)$.

\subsection{Doppler shift vs. line intensity}

Figure 6 shows Ne VIII Doppler shift vs. Ne VIII line intensity. For the equatorial region, once again two different trends are observed. The main part of the dispersion plot shows a clear decrease of the blue-shift with increasing Ne VIII line intensity, while in the low intensity range the blue-shift increases with increasing intensity. Once again no major differences are observed between the quiet Sun and coronal hole either in the position of maximum blue-shift or in the slope.

The decrease of the blue-shift with increasing Ne VIII line intensity is also clearly observed for the polar coronal hole, but does not appear in the quiet Sun where the slope is $a=0.4$. Again the contrast in the polar region is smaller than in the equatorial region which prevents us from obtaining any information about the very low or the very high intensity range for the polar region.

\subsection{Example of line shifts across the network}

The correlations shown in Figs. 5a and 5b reveal that statistically the maximum outflow does not take place at network centre (intensity maximum) but rather near its boundaries, i.e. intensities slightly above the median level. This pattern can be also found with individual examples.

We show an example of the variation of Ne VIII line shift across the network in the lower middle panel in Fig. 2. We defined a box stretching from one cell centre to a neighboring centre crossing a network patch and calculated the mean variation of Ne VIII line shift and filtered continuum intensity along the box giving the mean variation across the respective network patch. This network patch was chosen in quiet Sun, in a region were no strong magnetic concentration appears in the MDI magnetogram in order to rule out obvious coronal loop flows.
Generally we find the blue-shifts in the network to be stronger than in the inter-network, as already mentioned by Hassler et al. (1999). A closer inspection, however, shows a more complex structure: the Doppler shifts in the centre of the network are smaller than at the edges of the bright network patch. The maximum of the blue-shift appears near network boundaries rather than at network centre - directly above the centre of the network patch the Doppler shift of the coronal line is about zero. This behavior can be found with many network patches, even though there are exceptions to the rule.

In this study, we carefully avoided any threshold methods in investigating the relation of (continuum) intensity and coronal Doppler shifts, as these are notoriously subjective. Thus we are not able to give any percentage of coincidence for the latter example. Nevertheless, one might plot areas of large outflow on the filtered continuum images in order to get a general idea of the correlation between those regions that have the strongest blue-shift and the network boundaries. In Fig. 7, white patches represent areas of large blue-shifts in both polar and equatorial regions. In order to keep the figure as clear as possible, the "large blue-shifts" were defined differently according to the region. The blue-shifts that were selected for the polar region were those larger than $5 \mathrm{~km} \mathrm{~s}^{-1}$ for the quiet Sun, and those larger than $8 \mathrm{~km} \mathrm{~s}^{-1}$ for the coronal hole. For the equatorial region, those blue-shifts larger than $9 \mathrm{~km} \mathrm{~s}^{-1}$ for the quiet Sun were chosen, as well as those larger than $15 \mathrm{~km} \mathrm{~s}^{-1}$ for the coronal hole. One can clearly see that regions of large blue-shift mostly appear at network boundaries rather than at the network centre for the equatorial region (right panel in Fig. 7), although this phenomenon is not as clear in the polar region (left panel in Fig. 7). These results are only qualitative, although they do support the statistical results. This is why the statistical analysis in Sects. 4.3 and 4.4 is of vital importance.

\section{Discussion}

\subsection{Coronal flows and network structure}

At least two mechanisms can explain the pattern pointed out in Sects. 4.3 and 4.5, namely that the Ne VIII line shifts are strongest at the edges of the network patches and not in the centre.

One possibility might be that bipolar fields being swept in from the cell interior cause enhanced magnetic activity with the more or less monopolar network fields. This could lead to higher line shifts.

An alternative interpretation is a coronal funnel flow. Aiouaz et al. (2004) showed in a time-dependent MHD model including spectral line synthesis that the outflow across a coronal funnel is not trivial. Indeed the maximum of the outflow velocity is not reached above the funnel centre but rather in the vicinity of the centre mainly due to the magnetic configuration of the funnel.

\subsection{Coronal outflows}

The results in the main part of the dispersion plots in Figs. $6 \mathrm{a}, \mathrm{b}$ and $\mathrm{d}$ are in agreement with previous work done by 

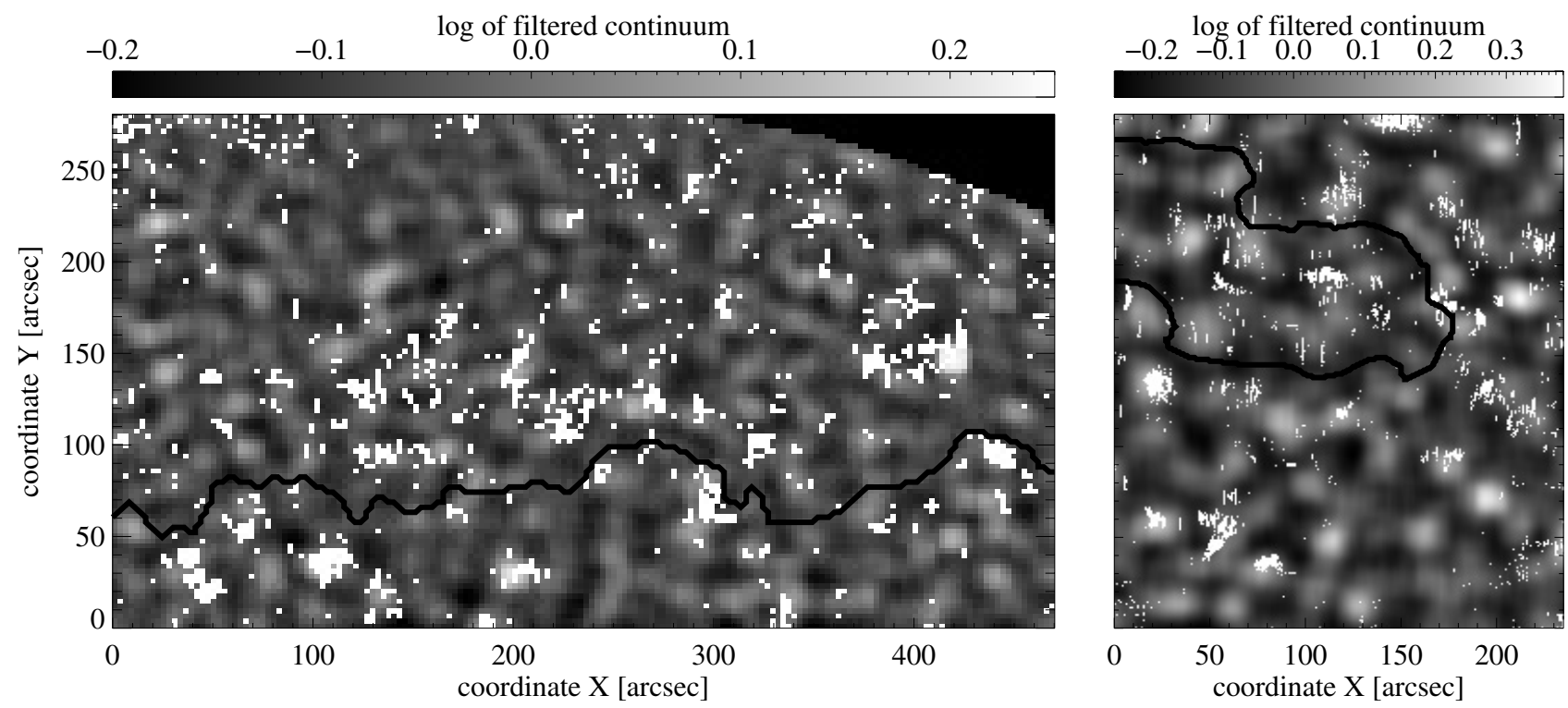

Fig. 7. Filtered continuum intensity images on logarithmic scale with white patches showing regions with the highest blue-shifts. Left: polar region with "highest blue-shifts" defined as those larger than $5 \mathrm{~km} \mathrm{~s}^{-1}$ for the quiet Sun, and larger than $8 \mathrm{~km} \mathrm{~s}^{-1}$ for the coronal hole. Right: equatorial region with blue-shifts larger than $9 \mathrm{~km} \mathrm{~s}^{-1}$ for the quiet Sun, and larger than $15 \mathrm{~km} \mathrm{~s}^{-1}$ for the coronal hole. The coronal hole is outlined with a black solid line. The highest blue-shifts clearly cluster along the network boundaries for the equatorial region; this result is not as obvious for the polar region.

Wilhelm (2000) using the same data set (data set 2). Wilhelm (2000) described in contour plots that the strongest blue-shift appears mostly in the dark region in Ne VIII line intensity. The increase of Ne VIII line shifts with decreasing Ne VIII line intensity, i.e. the fastest outflows found in the dark regions in Ne VIII line intensity, can be related to the energy balance: energy not lost through radiation is used to accelerate plasma in the corona. In other words, what goes into flow is not available for radiation.

A new result from the study presented here is that for very low intensities the equatorial region shows decreasing Doppler shifts with decreasing line intensity. At the lowest intensities (less than $20 \%$ of the median) the Doppler shifts are vanishing (see Figs. 6a and 6b). The interpretation could be that for very low intensity regions there is not enough energy available to either accelerate the wind or to produce detectable radiation.

\subsection{Relation to the magnetic field}

The results given in the preceeding sections show that one can distinguish different ranges of intensity by their relation to the Ne VIII Doppler shifts. Regions with filtered continuum intensities above approximately $25 \%$ of the average intensity $(\log (I /\langle I\rangle) \geq 0.25)$ and regions with Ne VIII line intensities below approximately half the average intensity $(\log (I /\langle I\rangle) \leq$ -0.40 ) show opposite signs of the slope in the line shift vs. intensity relation. To investigate the role of these two regions we compare them to the reconstructed magnetogram.

Figure 2 shows the Ne VIII line intensity (lower left panel), the filtered continuum intensity (upper middle panel) and the reconstructed magnetogram (upper right panel) based on
MDI/SOHO data. The coronal hole is outlined by the thick black line in the intensity images. Overplotted as thin black lines (upper middle and upper right panels) are contours of the filtered continuum intensity image for a value of 0.25 , i.e. the relative intensity that distinguishes the two regions of opposite sign for the line shift-intensity relation (see Figs. 5a and 5b). Overplotted as white lines (upper right and lower right panels) are contours of the Ne VIII line intensity image for a value of -0.40 (see Figs. 6a and 6b), the value that distinguishes the main part of the dispersion plot from the lowest intensities where the Doppler shifts are vanishing.

Generally the highest filtered continuum intensity (thin black contours) are associated with high magnetic field concentrations. This can be expected since the relation between bright network patches and magnetic field concentration has been known since Kopp \& Kuperus (1968). However these network patches do not necessarily coincide with the brightest regions in line intensity and not even necessarily with the brightest regions in the continuum image (upper left panel in Fig. 2). Furthermore these black contours seem to appear equally in quiet Sun and coronal hole areas. Since at the chromospheric level there is no signature of a coronal hole it explains why no fundamental differences are found between the quiet Sun and coronal hole in Fig. 5.

The white contours in the Ne VIII emission line image show that the very low intensity range comes mostly from the coronal hole or nearby. The same contour plot on the magnetogram shows no sign of magnetic field concentration. This lack of magnetic energy seems to confirm the interpretation in Sect. 5.2 which characterizes these regions as having a lack of energy to either accelerate the plasma or produce radiation. 


\section{Conclusion}

The main goal of this paper was to determine correlation between the network, Ne VIII line intensity and Ne VIII line shift.

The SUMER data presented above show clearly for the first time that the maximum outflow does not appear in the network centre nor especially at the intersection of network lanes (Hassler et al. 1999), but rather near network boundaries. These results are consistent with a recent 2D-MHD model (Aiouaz et al. 2004) and observations (Popescu et al. 2004). Furthermore we find similar properties of the coronal Doppler shifts in an equatorial coronal hole as previously published for a polar coronal hole (Wilhelm 2000). For the first time we establish that in the regions of very low coronal line intensities in coronal holes (lower than $40 \%$ of the average line intensity) the Doppler shifts tend to be zero. Therefore we conclude that there the energy present at coronal temperatures seems to be insufficient to either accelerate the plasma or to create detectable radiation. This interpretation is supported by the complete absence of magnetic field concentrations in these darkest regions of coronal holes.

At this point we can only speculate on how the magnetic field, the network, the line intensity and the line shift are connected. Nevertheless recently some works (see Xia et al. 2003; Popescu et al. 2004) have tried to relate those components of the solar atmosphere.

This study motivates further work on correlations between hotter and cooler lines and has serious implications on transition region and coronal funnel modeling as well as on our understanding of the acceleration of the solar wind and the structure of the solar atmosphere.

Acknowledgements. We would like to thank Reiner Hammer for the careful reading of the manuscript and for helpful comments. The SUMER project is financially supported by DLR, CNES, NASA, and the PRODEX project (Swiss contribution). SUMER and MDI are part of SOHO, the SOlar and Heliospheric Observatory of ESA and NASA. We thank the MDI team for the availability of the magnetic field data.

\section{References}

Aiouaz, T., Peter, H., \& Keppens, R. 2004, SOHO 13 Waves, Oscillations and Small-Scale Transients Events in the Solar Atmosphere: A Joint View from SOHO and TRACE, ed. H. Lacoste, ESA SP-547, 375
Athay, R. G., Gurman, J. B., Henze, W., \& Shine, R. A. 1983, ApJ, 265, 519

Brekke, P., Hassler, D. M., \& Wilhelm, K. 1997, Sol. Phys., 349

Brynildsen, N., Fredvik, T., Maltby, P., et al. 1997, Fifth SOHO Workshop: The Corona and Solar Wind Near Minimum Activity, ed. A. Wilson, ESA SP-404, 263

Chae, J., Schühle, U., \& Lemaire, P. 1998, ApJ, 505, 957

Charbonneau, P. 1995, ApJS, 101, 309

Curdt, W., Kucera, A., Rybak, J., Schühle, U., \& Wöhl, H. 1997, Fifth SOHO Workshop: The Corona and Solar Wind Near Minimum Activity, ed. A. Wilson, ESA SP-404, 307

Dammasch, I. E., Hassler, D. M., Curdt, W., \& Wilhelm, K. 1999, Space Sci. Rev., 87, 161

Dere, K. P., Bartoe, J.-D. F., \& Brueckner, G. E. 1984, ApJ, 281, 870

Doschek, G. A., Bohlin, J. D., \& Feldman, U. 1976, ApJ, 205, L177

Gabriel, A. H. 1976, Phil. Trans. R. Soc. Lond. A., 281, 339

Gallagher, P. T., Phillips, K. J. H., Harra-Murnion, L. K., \& Keenan, F. P. 1998, A\&A, 335, 733

Gebbie, K. B., Hill, F., November, L. J., et al. 1981, ApJ, 251, L115

Griffiths, N. W., Fisher, G. H., Woods, D. T., \& Siegmund, O. H. W. 1999, ApJ, 512, 992

Hassler, D. M., Dammasch, I. E., Lemaire, P., et al. 1999, Science, 283, 810

Judge, P. G., Hansteen, V., Wikstol, O., et al. 1998, ApJ, 502, 981

Kopp, R. A., \& Kuperus, M. 1968, Sol. Phys., 4, 212

Lemaire, P., Wilhelm, K., Curdt, W., et al. 1997, Sol. Phys., 170, 105

Lemaire, P., Bocchialini, K., Aletti, V., Hassler, D., \& Wilhelm, K. 1999, Space Sci. Rev., 87, 249

Patsourakos, S., Vial, J.-C., Gabriel, A. H., \& Bellamine, N. 1999, ApJ, 522, 540

Pauluhn, A., Solanki, S. K., Rüedi, I., Landi, E., \& Schühle, U. 2000, A\&A, 362

Peter, H. 1999, ApJ, 516, 490

Peter, H., \& Judge, P. G. 1999, ApJ, 522, 1148

Popescu, M. D., Doyle, J. G., \& Xia, L. D. 2004, A\&A, 421, 339

Reeves, E. 1976, Sol. Phys., 46, 53

Reeves, E. M., Foukal, P. V., Huber, M. C. E., et al. 1974, ApJ, 188, L27

Scherrer, P. H., Bogart, R. S., Bush, R. I., et al. 1995, Sol. Phys., 162, 129

Warren, H. P., Mariska, J. T., \& Wilhelm, K. 1997, ApJ, 490, L187

Wilhelm, K. 2000, A\&A, 360, 351

Wilhelm, K., Curdt, W., Marsch, E., et al. 1995a, Sol. Phys., 162, 189

Wilhelm, K., Curdt, W., Marsh, E., et al. 1995b, Proc. SPIE, 2517, 2

Xia, L. D., Marsch, E., \& Curdt, W. 2003, A\&A, 399, L5 